\title{
Vein Wrapping Technique for Nerve Reconstruction in Patients with Thyroid Cancer Invading the Recurrent Laryngeal Nerve
}

\author{
Young Moon Yoo, Il Jae Lee, Hyoseob Lim, Joo Hyoung Kim, Myong Chul Park \\ Department of Plastic and Reconstructive Surgery, Ajou University Hospital, Ajou University School of Medicine, Suwon, Korea
}

\begin{abstract}
Recurrent laryngeal nerve paralysis is the most common and serious complication after thyroid cancer surgery. The objective of this study was to report the advantages of the vein wrapping technique for nerve reconstruction in patients with thyroid cancer invading the recurrent laryngeal nerve and its effects on postoperative phonatory function. The subjects were three patients who underwent resection of the recurrent laryngeal nerve during surgical extirpation of papillary thyroid cancer. Free ansa cervicalis nerve graft or direct neurorrhaphy with a vein wrapping technique was used to facilitate nerve regeneration, protect the anastomosed nerve site mechanically, and prevent neuroma formation. Oneyear postoperative laryngoscopic examination revealed good vocal cord mobility. Maximum phonation time $(19.5 \pm 0.3 \mathrm{sec})$ was longer than a previously-reported value in conventional reconstruction patients $(18.8 \pm 6.6 \mathrm{sec})$. The present phonation efficiency index $(7.88 \pm 0.78)$ was higher than that previously calculated in conventional reconstruction $(7.59 \pm 2.82)$. The mean value of the Voice Handicap Index- 10 was 6 , which was within the normal range. This study demonstrates improvement in phonation indices measured 1 year after recurrent laryngeal nerve reconstruction. Our results confirm that the vein wrapping technique has theoretical advantages and could be favored over conventional reconstruction techniques for invenerate nerve injuries.
\end{abstract}

Keywords Recurrent laryngeal nerve / Thyroid neoplasms / Nerve regeneration / Phonation / Vocal cords

\author{
Correspondence: Myong Chul Park \\ Department of Plastic and \\ Reconstructive Surgery, Ajou \\ University Hospital, Ajou University \\ School of Medicine, 164 Worldcup-ro, \\ Yeongtong-gu, Suwon 443-721, Korea \\ Tel: $+82-31-219-5614$ \\ Fax: +82-31-219-5610 \\ E-mail:mpark@ajou.ac.kr \\ Co-Correspondence: Hyoseob Lim
}

This article was presented as a poster in the 68th Congress of the Korean Society of Plastic and Reconstructive Surgeons, on November 4-7, 2010 in Seoul, Korea and 6th Congress of the World Society for Reconstructive Microsurgery, on June 29-July 2, 2011 in Helsinky, Finland.

No potential conflict of interest relevant to this article was reported.

\section{INTRODUCTION}

Recurrent laryngeal nerve paralysis is the most common and serious complication after thyroid cancer surgery [1]. Initial postoperative recurrent laryngeal nerve paralysis has been reported with an incidence of $4 \%$ to $8 \%$; with continued follow-up, the recurrent laryngeal nerve frequently recovers its function, resulting in a permanent paralysis rate of $1 \%$ to $2 \%$ [2]. Recurrent laryngeal nerve paralysis causes hoarseness, shortening of phonation, aspiration, and increased vocal effort $[3,4]$. These symptoms adversely affect quality of life [3].

Several methods of severed recurrent laryngeal nerve reconstruction have been reported. If the defect is extremely short, the ends of the severed recurrent laryngeal nerve can be directly 
anastomosed without tension. However, if the defect is too long for direct neurorrhaphy, a free nerve graft taken from the transverse cervical nerve, supraclavicular nerve or ansa cervicalis nerve is used to fill the defect.

Secure nerve anastomosis is the most important in successful nerve reconstruction [5]. In particular, there are two anastomosis sites when free nerve grafts are applied, so proper technique is needed. Several techniques for enhancement of nerve recovery after nerve anastomosis have been developed. Feasible approaches include tubulization with biological or synthetic materials as graft conduits and the epineural sleeve technique [5-8].

In our study, defects of the severed recurrent laryngeal nerve were anastomosed directly or filled with a free ansa cervicalis nerve graft immediately after extirpation of thyroid cancer. We designed a special vein wrapping technique to enhance nerve recovery. We report here the advantages of the vein wrapping technique for nerve reconstruction in patients with thyroid cancer invading the recurrent laryngeal nerve and its effects on postoperative phonatory function.

\section{IDEA}

The subjects were three patients (all female, mean age 51 years; range, 44 to 59 years) with advanced thyroid cancer who underwent resection of the recurrent laryngeal nerve during surgical extirpation of the thyroid cancer in February and March, 2010. All patients were diagnosed with papillary thyroid cancer by means of histological examination of specimens (Table 1). There were two cases in which a free ansa cervicalis nerve graft was used to reconstruct the recurrent laryngeal nerve after tumor extirpation. They were too long to permit direct neurorrhaphy ( $3 \mathrm{~cm}$ long nerve defect in patient 1 and $4 \mathrm{~cm}$ long in patient 3). Upon exploration, the left ansa cervicalis nerve was intact, so that the nerve was harvested as a 4-cm-long segment. Free nerve grafting on the proximal end was done with \#9-0 nylon using the epineural suture technique. The left anterior jugular vein was harvested and wrapped around the proximal anastomosis site. Then, distal end anastomosis was performed

Table 1. Patient data
\begin{tabular}{|lccc|}
\hline \multicolumn{4}{|c|}{} \\
\hline Characteristics & Patient 1 & Patient 2 & Patient 3 \\
\hline Sex & F & F & F \\
Age (yr) & 50 & 44 & 59 \\
Tumor pathology & PTC & PTC & PTC \\
Lymph node metastasis & $(-)$ & $(+)$ at 3/66 & $(+)$ at 5/45 \\
Distant metastasis & $(-)$ & $(-)$ & $(-)$ \\
Nerve defect length (cm) & 3 & 1 & 4 \\
\hline PTC, papillary thyroid cancer. & & & \\
\hline
\end{tabular}

with vein wrapping using the same method (Fig. 1A). In case of patient 2, a 1-cm-long right recurrent laryngeal nerve defect due to tumor extirpation was noted. Direct neurorrhaphy was performed with \#9-0 nylon using the epineural suture technique because the defect was short. The right anterior jugular vein was harvested and wrapped around the anastomosis site (Fig. 1B).

To assess vocal cord function, laryngoscopic examinations were performed periodically after the operation (short-term follow-up at 3 months after the operation and long-term followup 1 year postoperatively). Also, the maximum phonation time (MPT) and phonation efficiency index (PEI) determined 1 year postoperatively were used to objectively evaluate the outcome. MPT is the length of sustained phonation of the vowel "a" at the loudness of a usual conversational voice after maximum inspiration in a sitting position [9]. The ratio of MPT and vital capacity (VC; sec/L)-which should indicate vocal cord function by converting a unit volume of exhaled air to certain duration of phonation-was calculated. Thus, this value is called the PEI [9]. For the evaluation of the patient's subjective concerns, we applied the Voice Handicap Index-10 (VHI-10), adapted from Rosen et al. [10]; 2004 obtained 1 year after the operation. The

\section{Fig. 1. Illustration of the vein wrapping technique}

(A) Free ansa cervicalis nerve graft with vein wrapping technique. (B) Direct neurorrhaphy with vein wrapping technique.

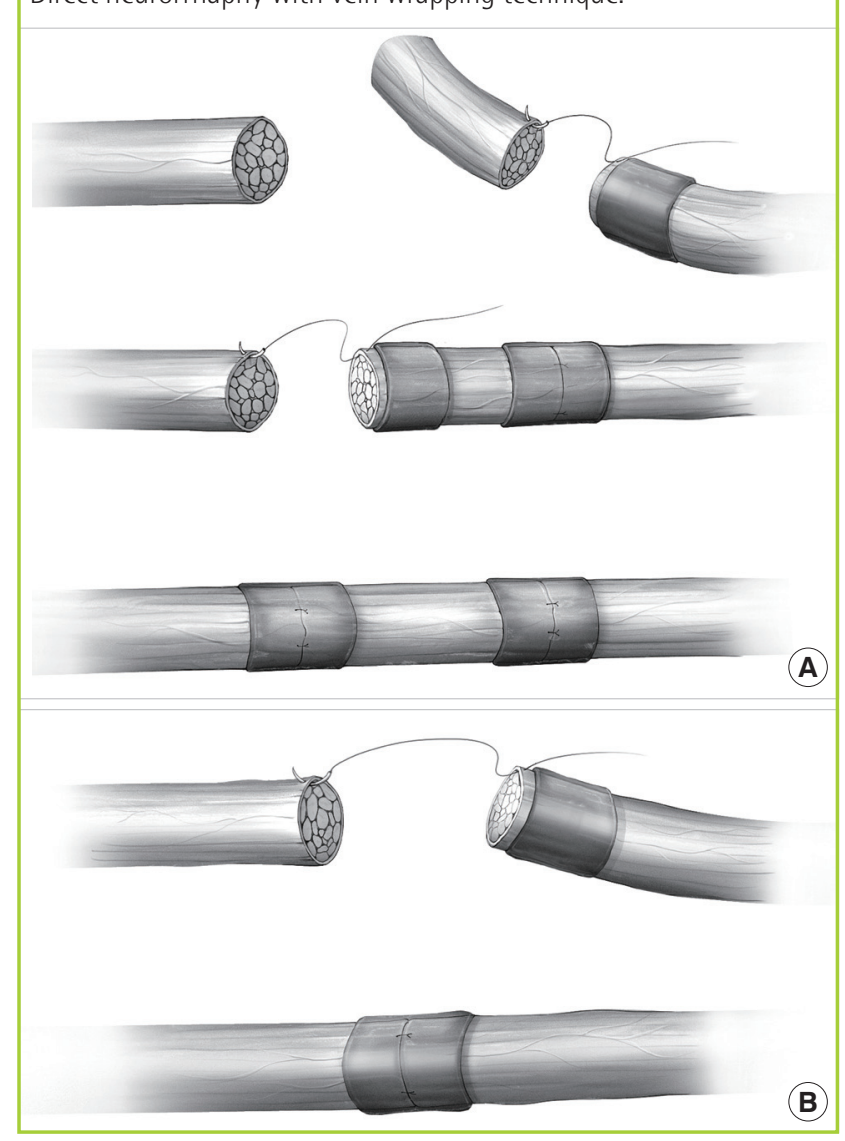


VHI-10 comprises 10 questions from the original 30 questions of the VHI [11].

Laryngoscopic examination conducted as a short-term followup at 3 months after the operation showed that the vocal cords on that side were fixed in the median, but had good tension during phonation and that the gaps between the cords were narrow during phonation [9]. Although patient $3 \mathrm{did}$ not participate in the long-term follow-up, the other two patients who did showed a better vocal cord mobility at 1 year than at 3 months postop- eratively (Fig. 2). MPT and PEI at 1 year postoperatively was $19.5 \pm 0.3 \mathrm{sec}$ and $7.88 \pm 0.78 \mathrm{sec}$, respectively. The mean value of VHI-10 was 6 (Table 2). There were no major complications such as infection, hematoma, or recurrence of cancer.

\section{DISCUSSION}

The recurrent laryngeal nerve is an essential nerve for phonation and psychosocial interaction [12]. Therefore, when the recur-

\section{Fig. 2. Photography of patient 2}

(A) Direct neurorrhaphy was performed with \#9-0 nylon. (B) Vein wrapping was performed with the right anterior jugular vein. (C) Laryngoscopic findings during maximal inspiration (3 months after operation). (D) Laryngoscopic findings during phonation (3 months after operation). (E) Laryngoscopic findings during maximal inspiration (1 year after operation). (F) Laryngoscopic findings during phonation (1 year after operation).
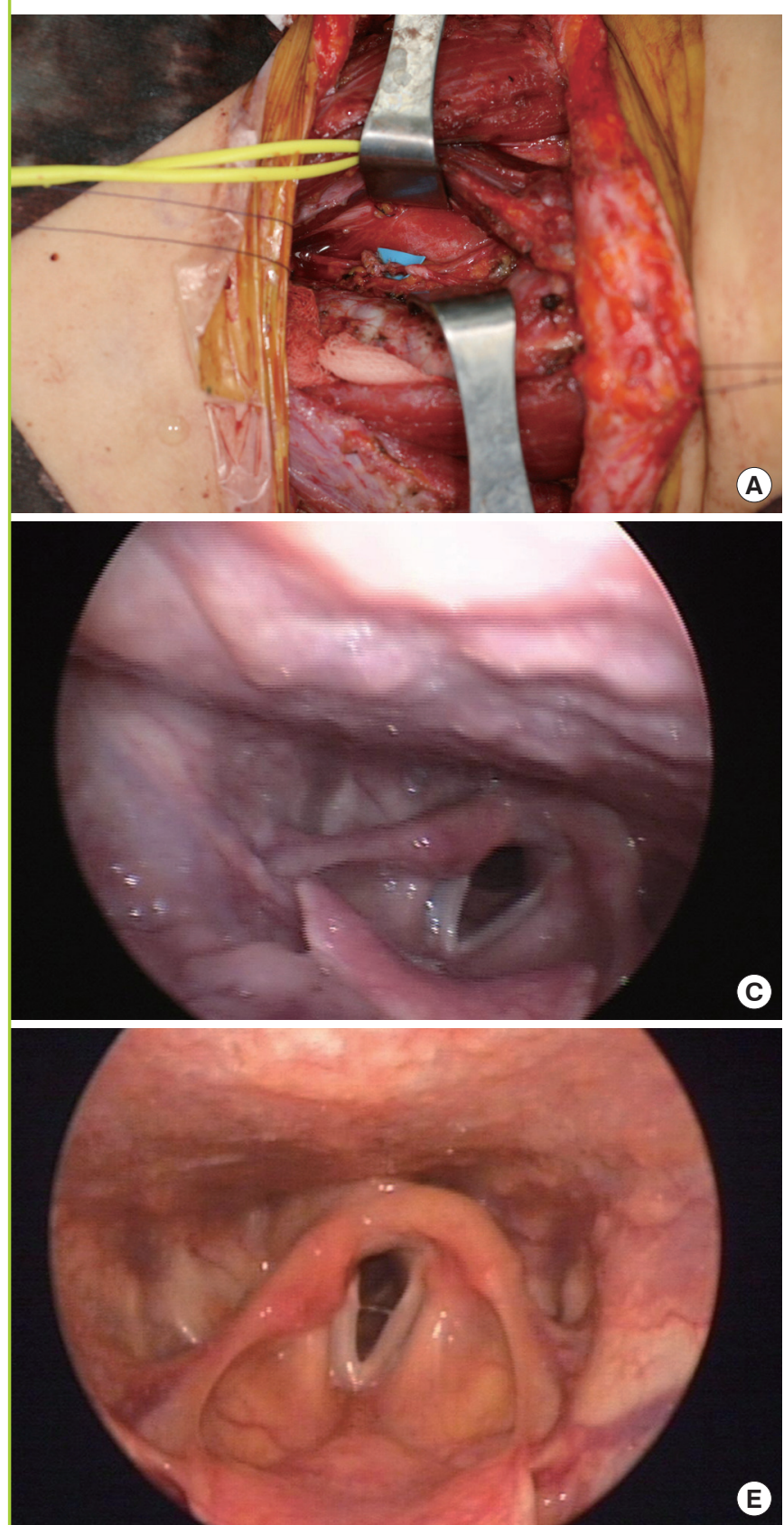
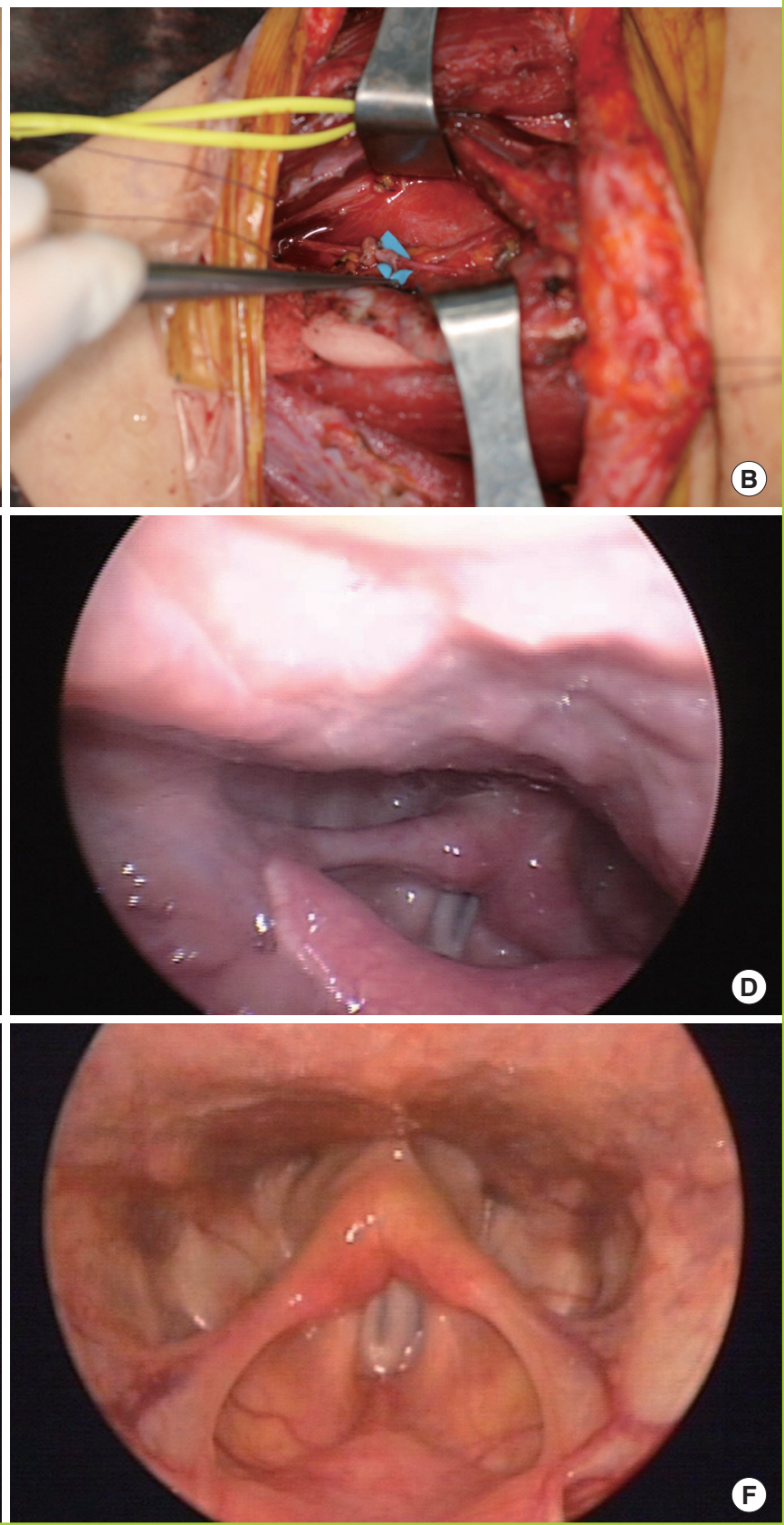


\begin{tabular}{|c|c|c|c|}
\hline & Patient 1 & Patient 2 & Patient 3 \\
\hline $\begin{array}{l}\text { Laryngoscopic finding } \\
\text { (vocal cord paralysis) }\end{array}$ & Left & Right & Left \\
\hline $\begin{array}{l}\text { Maximum phonation time (sec) } \\
\text { at } 1 \text { yr after operation }\end{array}$ & 19.3 & 19.7 & - \\
\hline $\begin{array}{l}\text { Phonation efficiency index (sec/L) } \\
\text { at } 1 \text { yr after operation }\end{array}$ & 8.43 & 7.32 & - \\
\hline $\begin{array}{l}\text { Voice Handicap Index-10 (score) } \\
\text { at } 1 \text { yr after operation }\end{array}$ & 6 & 6 & - \\
\hline
\end{tabular}

rent laryngeal nerve is injured, immediate reconstruction with direct neurorrhaphy or a free nerve graft is important to preserve its function [12].

We used the vein wrapping technique because of its theoretical advantages. The first advantage involves facilitating nerve regeneration. The anastomosis site becomes separated from the surrounding tissues, supplying an optimal environment for nerve regeneration. The technique was designed to enhance regeneration of the nerve graft by creating a biological chamber at the anastomosis site that would collect axoplasmic fluid from the transected nerve ends [5]. The second advantage relates to the mechanical protection of the anastomosed nerve site. A mechanical chamber could prevent protrusion of fascicles out from the suture line and sprouting axons can be well-aligned within the chamber [5]. The third advantage concerns prevention of neuroma formation. When nerve transection injury occurs, proximally regenerating axons sprout into the surrounding tissue and may grow aberrantly, forming dense nerve tangles called neuromas [13]. To prevent neuroma formation, the vein wrapping technique can be done with the goal of isolating it from the inflammatory cascade and neurotrophic factor production triggered by nerve trauma in the surrounding tissues [13].

Veins potentially available for nerve wrapping are easily accessible and available in the same operative field. Veins can be easily dilated with fine surgical forceps to adapt the lumen to the nerve size and can thus be used to treat large nerves [13]. Although successful wrapping with numerous synthetic materials (such as silicon and collagen conduits) has been reported, veins have the advantage of being obtainable at no cost and are easily available. Furthermore, this technique does not cause any risk of venous thrombosis [13].

In the postoperative course, recurrent laryngeal nerve and vocal cord function can be examined by a variety of means: self assessment of voice, nerve stimulation, cricothyroid palpation, or the use of proper equipment for direct visualization. A direct laryngoscopy can be used to check vocal cord mobility; however, it is not by itself sufficient to evaluate the recovery of vocal cord function, as the reinnervated cords are usually fixed in the median [4]. For more exact evaluation of the recovery of the voice quantitatively, the MPT and PEI were measured. In this study, subjective assessment was performed on all patients postoperatively by the VHI-10 [11]. Self assessment also provides different information about voice function, so the VHI-10 can be useful to evaluate the vocal cord function.

We had few patients and no control group, so our results were compared with the data from a previous study [9]. Patients with thyroid cancer underwent reconstruction of the recurrent laryngeal nerve. The recurrent laryngeal nerve was reconstructed with several conventional techniques such as direct neurorrhaphy, free nerve graft, ansa cervicalis nerve-recurrent laryngeal nerve anastomosis, and vagus nerve-recurrent laryngeal nerve anastomosis without the vein wrapping technique [9].

Our study showed that 1 year postoperatively, the patients displayed longer MPT $(19.5 \pm 0.3 \mathrm{sec})$ than the previously-reported [9] conventional reconstruction group (18.8 $\pm 6.6 \mathrm{sec}$ ). Also, the present patients displayed a higher PEI $(7.88 \pm 0.78)$ than the previous patients $(7.59 \pm 2.82)$ [9]. A very recent study reported that a VHI-10 score $>7$ should be considered abnormal. In our study, the mean value of the VHI-10 was 6, so it was within the normal range.

This series is limited by the drawbacks of a retrospective design and few study cases. Furthermore, there was no control group, thereby exposing this series to selection bias. However, this study used a similar design and similar patient groups to a prior study so that the results could be compared [9].

\section{REFERENCES}

1. Chiang FY, Lu IC, Kuo WR, et al. The mechanism of recurrent laryngeal nerve injury during thyroid surgery: the application of intraoperative neuromonitoring. Surgery 2008; 143:743-9.

2. Snyder SK, Lairmore TC, Hendricks JC, et al. Elucidating mechanisms of recurrent laryngeal nerve injury during thyroidectomy and parathyroidectomy. J Am Coll Surg 2008; 206:123-30.

3. Yumoto E, Sanuki T, Kumai Y. Immediate recurrent laryngeal nerve reconstruction and vocal outcome. Laryngoscope 2006; 116:1657-61.

4. Miyauchi A, Matsusaka K, Kihara M, et al. The role of ansato-recurrent-laryngeal nerve anastomosis in operations for thyroid cancer. Eur J Surg 1998;164:927-33.

5. Lubiatowski P, Unsal FM, Nair D, et al. The epineural sleeve technique for nerve graft reconstruction enhances nerve recovery. Microsurgery 2008;28:160-7. 
6. Battiston B, Geuna S, Ferrero M, et al. Nerve repair by means of tubulization: literature review and personal clinical experience comparing biological and synthetic conduits for sensory nerve repair. Microsurgery 2005;25:258-67.

7. Heijke GC, Klopper PJ, Baljet B, et al. Silicone rubber tubulization in peripheral sensory nerve reconstruction: an experimental study in rabbits. Microsurgery 2001;21:306-16.

8. Meyer RS, Abrams RA, Botte MJ, et al. Functional recovery following neurorrhaphy of the rat sciatic nerve by epineurial repair compared with tubulization. J Orthop Res 1997; 15:664-9.

9. Miyauchi A, Inoue H, Tomoda C, et al. Improvement in phonation after reconstruction of the recurrent laryngeal nerve in patients with thyroid cancer invading the nerve. Surgery 2009;146:1056-62.

10. Rosen CA, Lee AS, Osborne J, et al. Development and validation of the voice handicap index-10. Laryngoscope 2004;114:1549-56.

11. Arffa RE, Krishna P, Gartner-Schmidt J, et al. Normative values for the voice handicap index-10. J Voice 2011 Aug 3 [Epub]. http://dx.doi.org/10.1016/j.jvoice.2011.04.006.

12. O’Neill JP, Fenton JE. The recurrent laryngeal nerve in thyroid surgery. Surgeon 2008;6:373-7.

13. Galeano M, Manasseri B, Risitano G, et al. A free vein graft cap influences neuroma formation after nerve transection. Microsurgery 2009;29:568-72. 